NOTAS Y DISCUSIONES

\title{
Sobre el concepto de igualdad: algunas observaciones
}

\author{
ISABEL SANTA CRUZ
}

Universidad de Buenos Aires

El feminismo contemporáneo, como sabemos, dista de ser homogéneo. Todo feminismo, por cierto, denuncia la sujeción de las mujeres, subraya su injusticia, tiene la convicción de la posibilidad de modificar la situación y la voluntad compartida de superar la estructura de dominación que afecta a la diferencia de sexos.' Podemos decir, de un modo general, con Monique Remy, que "consideramos como feminista toda palabra, todo escrito, o todo movimiento relativo a la condición de las mujeres en la sociedad, si denuncia esta condición como resultado de una relación de dominación de un sexo (masculino) sobre el otro (femenino) ${ }^{2}$ A pesar de este denominador común, hay dentro del feminismo divergencias importantes en las líneas teoricas como en las estrategias prácticas. No hay, pues, una teoría general del feminismo. En todos los grupos que, desde hace mucho tiempo, reivindican la emancipación de las mujeres operan temáticas emparentadas y próximas, pero no idénticas. A lo sumo, dice M. Remy, «el feminismo podría ser definido como un conjunto de doctrinas que poseen niveles de estructuración muy diversos».3 Más aún, en los últimos años asistimos a la constitución de grupos y subgrupos que resisten todo intento de clasificación. Sin embargo, como afirma Rosi Braidotti, wen este tejido complejo, polivalente, de grupos productores de prácticas discursivas, podemos relevar dominios de preocupaciones comunes, coaliciones de intereses que hacen del movimiento una máquina política gobernada por la voluntad compartida por todas de mejorar el estatus de las mujeres ${ }^{4}{ }^{4}$

Parece innegable que, para mejorar el estatus de las mujeres, lograr su igualdad es un objetivo válido y valioso del pensamiento y de la acción feministas. Exigir la igualdad, entendida ésta como igualdad formal, como igualdad ante la ley, como igualdad de oportunidades en lo que toca a educación, empleo, propiedad de bienes, paga por trabajo y, en general, todo lo referente a derechos humanos, es inobjetable en nombre del universalismo y de la justicia. ${ }^{5}$ Pero está claro que la igualdad para las mujeres de la que se trata no es una igualdad de las mujeres entre si, sino una igualdad entre los sexos, una igualdad de las mujeres con los varones. $Y$, en este aspecto, la igualdad como objetivo presenta algunas aris- 
tas problemáticas. En efecto, la crítica fundamental que se hace a las posiciones igualitaristas ${ }^{\circ}$ es que ellas acaban por reforzar la estructura de dominación de la sociedad patriarcal, puesto que buscan que las mujeres sean, piensen y actúen como los hombres. El igualitarismo - se dice- parece aceptar, y aún aplaudir, el modelo masculino y buscar para las mujeres una asimilación, una integración, que no es sino homologación, cooptación, conformación a un paradigma androcéntrico disfrazado de neutro universal. Desde tal perspectiva, la igualdad no consistiría, pues, sino en una absorción en la masculinidad como parámetro de igualdad y el simple igualitarismo sería sólo una manera de convalidar el sexismo. El problema del igualitarismo, entonces, es que conduce a crear una sociedad sexualmente neutra $y$ una moralidad universal que de hecho implica una inmersión de la diferencia en lo masculino.?

Debe reconocerse que apelar a la mera igualdad formal es insuficiente. ${ }^{8}$ Porque la igualdad, ese concepto que, junto con sus dos socias, la libertad y la fraternidad, nos legó la Revolución francesa, no es identidad. La equiparación de igualdad con identidad es una huella impresa por el pensamiento de la Ilustración, para el que la igualdad pasa por la identidad: no se puede ser humano sino de una única manera; y la asimilación es siempre pensada como identificación con el modelo dominante. Bajo el manto del universalismo, el modo de volverse plenamente humana para la mujer es volviéndose hombre (o imitándolo)."

En este trabajo quiero presentar algunos elementos que ayuden a precisar y a acotar el concepto de igualdad. Intentaré mostrar en qué sentido la igualdad que se demanda para las mu- jeres es mucho más compleja que una igualdad formal y señalaré luego algunos problemas que ineludiblemente deben enfrentar las mujeres cuando la reivindican teóricamente y cuando intentan acceder a ella.

"Igualdad" se dice de muchas maneras, diría Aristóteles. Es un «discutiblen, diría Platón. No es, en efecto, un término univoco ni simple. Es un concepto abstracto no sustancializable, ${ }^{10}$ que bien puede usarse como una suerte de etiqueta simplista para encubrir más que para revelar. La igualdad es, ante todo, algún tipo de relación. Dejando, claro está, de lado su significación matemática y restringiéndonos al terreno político, debemos decir que, a pesar de las resonancias cuantitativas de la palabra, la igualdad no es un concepto cuantitativo: igualdad política nunca significa tener una misma cantidad ni los mismos grados de alguna característica, propiedad, función o derecho determinados." Para explicitar el significado de igualdad política me parece adecuado enfocarla como una relación de semejanza. Y esto me obliga a explicar que ha de entenderse entonces por «semejanza".

"Semejanza" es también polisémico, es otro adiscutible» que tiene, por lo menos, dos significados básicos:12

A. Semejanza es la relación entre dos o más términos que comparten uno o más caracteres idénticos, que son en ellos los mismos y respecto de los cuales se dice que los términos son semejantes entre sí. Esta es, pues, a) una semejanza rectproca, que se establece whorizontalmentew entre términos que pertenecen a un mismo nivel, y b) una semejanza sólo respecto de esa caracteristica o caracteristicas idénticas compartidas.

B. Semejanza, por otro lado, es la 
relación que se establece entre uno o más términos con otro término al que imitan y que les sirve de referente. Este tipo de semejanza ya no es reciproca sino unidireccional y "vertical». Supone que dos o más términos se asemejan a otro sin que éste, inversamente, se asemeje a ellos. Se trata de la clase de semejanza que se verifica entre la copia y su modelo, entre la imagen y su original. Los términos de la relación están en niveles diferentes y la semejanza comporta entonces una jerarquía, una relación de derivación y dependencia de los semejantes respecto de lo semejado.

La igualdad política debe entenderse como una relación de semejanza que responda al modelo A y no al modelo $B$. Una igualdad concebida sobre el modelo $\mathbf{B}$ cs, sin duda, pasible de las críticas a las que antes hicimos referencia, en la medida en que significaría una mera asimilación a un modelo ya dado como paradigma y nos permitiría decir, a la manera del Orwell de Rebelión en la granja, que todos los seres humanos son íguales, pero que hay algunos que son más iguales que otros.

Ahora bien, tomar la igualdad como una semejanza recíproca nos impone, al menos, dos exigencias: 1 . reconocer que los términos iguales, para poder serlo, deben ser diferentes entre sí, dado que la semejanza no es entre ellos una identidad absoluta, sino sólo respecto de algunos determinados caracteres y puede darse si $\mathrm{y}$ sólo si ellos son diferentes en otros múltiples caracteres; 2 . precisar cuáles son los caracteres mínimos que deben poseer idénticamente los iguales. Estas dos cuestiones que acabo de señalar están vinculadas entre sí. Dados los límites fijados al presente trabajo, no podré sino enumerar los caracteres implícitos en el conccpto de igualdad y esbozar su significado. Me contentaré, por lo demás, con senalar algunas dificultades que derivan de la primera exigencia que acabo de puntualizar.

La igualdad, entendida no como identidad ni uniformidad ni estandardización, ${ }^{13}$ comporta, en primer lugar, la autonomía, es decir, la posibilidad de elección y decisión independientes, que involucra la posibilidad de autodesignación. ${ }^{14}$ En segundo lugar, igualdad supone autoridad o, lo que es lo mismo, la capacidad de ejercicio de poder, el «poder poder», como dice $\mathrm{Ce}$ lia Amoró: : $^{15}$ "sólo puede llamarse iguales a quienes son equipotentes». En tercer lugar, y estrechamente conectada con. la equipotencia, la igualdad requiere lo que podríamos llamar equifonta, es decir, la posibilidad de emitir una voz que sea escuchada $y$ considerada como portadora de significado y de verdad, y goce, en consecuencia, de credibilidad. El cuarto carácter exigido por la igualdad es la equivalencia: tener el mismo valor, no ser considerado ni por debajo ni por encima de otro. ${ }^{16}$ No basta con poder, sino que también hay que valer, con la respetabilidad que ello implica.

Los caracteres señalados tienen por base necesaria la reciprocidad y el mutuo reconocimiento ${ }^{17}$ que pertenecen por definición a la relación de igualdad. Otras dos notas que en buena medida están implícitas en las que deslindamos son la interlocución, es decir, la situación en la que se dé una relación dialogica tal que sean todos interlocutores y ninguno "interlocutado"18 y la responsabilidad en el diseño y el mecanismo de la vida social. ${ }^{19} \mathrm{Y}$ al hablar de vida social no me refiero únicamente al terreno de lo político, sino a todo el entramado de relaciones interpersonales, incluyendo, claro está, las relaciones familiares y duales. ${ }^{20}$ 
La condición de posibilidad de la igualdad tal como la hemos delineado es que las mujeres sean, como los varones, sujetos, no en un sentido ontológico sino como quienes pueden "aparecer por la palabra y por la acción en el mundo público y privado, volverse actores, actrices del mundo común, actores que no puedan ser nunca confundidos con autores», para decirlo con palabras de Françoise Collin.. Cada uno de los iguales ha de ser un ego, un sí mismo, un self. ${ }^{22}$ La igualdad no se opera sino en el reconocimiento de la multiplicidad heterogénea de los ego, de que cada ego es una relación consigo mismo, que se prefiere a sí mismo, pero que reconoce a la vez que los demás también son ego. ${ }^{23}$

Recapitulando: la igualdad reivindicada para las mujeres es una relación no identificativa de semejanza recíproca, que comporta autonomía, equipotencia, equifonia, equivalencia, interlocución y responsabilidad de los individuos-sujetos actuantes en todas las relaciones sociales, familiares y duales. Pero para las mujeres la igualdad es una dura empresa. "Cada centínetro de igualdad cuesta», dice Amelia Val-

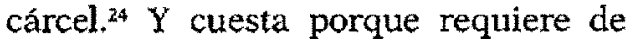
las mujeres una emergencia como sujeto $\mathrm{y}$, a la vez, una desidentificación con su genérico, colectivo de idénticas heterodesignado, como lo ha mostrado Celia Amoros. ${ }^{25}$ Los esquenas patriarcales no han hecho lugar para las mujeres en lo que a la relación individuocolectivo genérico se refiere. Para la mujer es problemático compaginar la voluntad de distinción personal, la autoafirmación, con la demanda de su genérico de "no ser distinta", de "ser como las otras». El orden simbólico social, diseñado masculinamente, parece no ofrecer salida a este problema ${ }^{26} y$ sume a las mujeres en una considera- ble ambigüedad o ambivalencia, que ha sido tematizada por más de una teóriça. ${ }^{27}$

El mundo masculino ha sido constituido como un espacio de iguales, esto es, como un cosmos estructurado, con sus reglas de juego, en el que cada uno tiene un puesto que ocupar. Las mujeres, como genérico colonizado $\mathrm{y}$ heterodesignado, fuera del pacto, ${ }^{28}$ han sido dejadas fuera del espacio. Están donde han sido puestas: en las margenes, en la regio dissimilitudinis. ${ }^{29}$ En este sentido, las mujeres como genérico son a-tópicas, no están en ningún puesto del espacio, sencillamente porque no han intervenido en su diseño y mecanismo. $X$ obtener un puesto en el espacio les exige hacerse a-típicas, alterarse..$^{30} \mathrm{El}$ acceso a la igualdad somete a la mujer a una tension, a una suerte de flexibilización de su identidad, a ser lo que podríamos llamar una «anfibia»: una individua que adopta el género posicionalmente. La posicionalidad en el género como acto de pensamiento y gesto de existencia ha sido caracterizada en un trabajo anterior, ${ }^{31}$ en el que intentamos mostrar que posicionarse en el género, elígiéndolo como punto de partida de prácticas y significados entre los otros múltiples ejes de identidad, es de importancia fundamental, en la medida en que la desigualdad sexual - para decirlo con palabras de Cristina Molina- es «la más ínsidiosa, más antigua y la más pertinaz de todas las desigualdadesm.32

Si para dejar de ser a-tópicas las mujeres deben volverse a-típicas sin olvidar, empero, el horizonte de identidad genérica, ese proceso exige que sean - permítaseme jugar con las palabras - en cierto modo u-tópicas. Y digo en cierto modo, porque no pretendo defender una utopía en el sentido de ideal que, por su propia perfec- 
ción, sería irrealizable y sólo perteneciente al dominio de la ciencia-ficción. ${ }^{33}$ La igualdad compleja que he caracterizado y que reivindicamos no es, por cierto, un objeto real, pero sí un objeto virtual, algo no existente aún como real pero sí existente como posible. ${ }^{34}$ Postular la igualdad como objeto virtual sirve, por lo menos, a dos propósitos: por un lado, nos permite analizar críticamente nuestro pasado y nuestro presente e interpretarlos; por otro, nos incita a planear estrategias y diseñar prácticas -incluidas las teóricas - tendentes a su realización. Plantear la igualdad como objeto virtual y como objetivo valioso no implica, sin embargo, ninguna definición ni de lo que las mujeres (y los varones) son ni de lo que deberian ser. ${ }^{35}$ No supone, tampoco, que la igualdad sea condición necesaria y suficiente de una sociedad moralmente buena. Supone solamente que la igualdad es condición de posibilidad para que pueda diseñarse $y$ edificarse un orden social fundado en el universalismo y la justicia. ${ }^{36}$

Ninguna emancipación de la mujer es posible si se realiza sólo en el plano individual y este es un principio en el que coinciden posturas muy divergentes entre sí. ${ }^{37}$ Pero al insistir en la necesidad de establecer relaciones entre mujeres para el logro de objetivos comunes no debemos disimular el hecho de que amujer" o "mujeres" son términos cuyo significado se restringe más y más, palabras cuya comprensión es ya mínima. ${ }^{38}$ Está bien criticar el carácter heterodesignado de "las mujeres" y sus connotaciones normativas. Pero por tratar de desposeer al varón del derecho de hablar de «las mujeres» y en nombre de ellas no debemos incurrir en el error de hablar, también nosotras, de todas las mujeres. Aun cuando pueda haber importantes puntos de contacto entre todas las mujeres, se dan entre ellas diferencias fundamentales que no pueden borronearse ni considerarse meramente irrelevantes o secundarias. Hoy ya no es posible, ni en el plano teórico ni en el político, hacer invisible o encubrir la complejidad de las diferencias culturales entre mujeres tras la cómoda etiqueta de una sororidad -biológica o estratégicaque nos uniría frente al "enemigo común». Las diferencias en razón de raza, religion, nacionalidad, clase social, posición económica, nivel cultural, sexualidad, tradición, etc., elementos todos ellos que son los ejes que conforman las identidades personales, son diferencias que marcan, separan, dividen y aun enfrentan a las mujeres entre sí. Son diferencias que interactúan y se entrecruzan, que se canalizan conjuntamente de diferentes maneras en diferentes contextos histórico-culturales. El aplastamiento de estas diferencias en nombre de una igualdad fundada en un mínimo denominador común es ya inadmisible, por perimido, y exige urgente y seria atención teórica. ${ }^{39}$

"Debemos tener el coraje de hacer frente a nuestras diferencias", dice Rosi Braidotti. ${ }^{40}$ Porque relevar y tematizar críticamente las diferencias entre las mujeres cuando se reivindica su igualdad no destruye la política feminista, sino que la complejiza y la enriquece tanto teórica como prácticamente. Si hemos sido siempre objeto de heterodesignación, si se nos ha tomado en bloque para pontificar sobre nosotras sin discernir entre nosotras, no cometamos el error de perpetuar los esquemas que heredamos: por haber sido víctimas de la "falacia de Protarco" 41 no incurramos, también nosotras, en ella. 
NOTAS

1. Cf. Linda Gordon: "What's New in Women's History*, en Teresa De Lauretis (ed.), $\mathrm{Fe}$ minist Studies. Critical Sizdies, Bloomington, Indiana University Press, 1986, p. 29 y Françoise Collin: «raxis de la difference. Notes sur le tragique du sujet», Provenances de la pensée. Femmes/Phitosophie, Les Cahiers du Grif, 46 (1992), p. 132 .

2. Monique Remy: De l'utopie d fintegration. Histoire des mouvements de femmes, Paris, Ed. L'Harmattan, 1990, p. 17.

3. Ibid.

4. Rosi Braidotti: "Thêories des études féministes, Quelques experiences contemporaines en Europe ${ }$, Savoir et difference des sexes, Les Cahiers du Grif. 45 (1990), p. 43.

5. La polemica igualdad-diferencia sigue ocupando un lugar importante dentro de los debates feministas actuales. Hay, por cierto, una variada gama de posturas teóricas entre quienes apoyan una reivindicación de la igualdad que lleva a neutralizar más de la cuenta las diferencias genericas y quienes exageran hasta el escandalo la diferencia, adoptando posiciones esencialistas y convirtiendo en fuertemente positivos los caracteres y funciones tradicionalmente asociados negativamente al genérico femenino.

6. Fundamentalmente a las igualitaristas liberales. Para el feminismo liberal of. M. Tapper: "Can a Feminist be a Liberal?" en Janna Thompson (ed). Women and philosophy. Australasian Association of Philosophy, La Trobe University, vol. 64 (1986), pp. $37-47$ y Susan Wendel: $A$ (Qualified) Defence of Liberalism», Hypatia, 2 (1987) 2, pp. 65-93.

7. Cf. Lorraine Code: Simply Equality is not Enoughn, en J. Thompson (ed.), op. cit., pp. 4865; Gatens, Moira: "Rousseau and Wollstonecraft: nature vs. Reason*, en J. Thompson (ed.), op, cit., p. 14-15 especialmente; Marilyn French: Beyond Power, On Women, Men \& Morals, Londres, Sphere Books, 1986, pp. 512-514; Dlana Coole: Women in Political Theory. From Ancient Misoginy to Contemporary Feminism, Worcester, Billing \& Sons, 1988, pp, 240-241; Alisa Del Re: *Pratiques politiques et binômes theoriques dans le ferminisme contemporain $*$, en Savoir et difference des sexes, op. cit., pp. 20-21; Rosi Braidotti: art. cit., p. 40.

8. Llama la atención que en el Diccionario ideologico feminista de Victoria Sau no figuren entre las palabras definidas ni "igualdad" ni adiferencias. Apenas se refiere a esia ultima al tratar el *feminismo de la diferencia ${ }_{\text {, }}$ cuyo origen. por lo demás, ubica erroneamente en 1978 , sin precisiones ulteriores. No son de gran ayuda tampoco las referencias sobre igualdad y diferencia inclutdas en The Dictionary of Femintist
Theory de Maggie Humm, Nueva York, Harvester, 1989.

9. Cf. F. Collin: art. cit, pp. 131-132; Mary Midgley: "On Not Being Afraid of Natural Sex Differences", en Morwenna Griffiths y Margaret Whitford (eds.), Feminist Perspectives in Philosophy, Bloomington, Indiana University Press, 1988, p. 34

10. Cf. M. French: op. cit., p. 525 .

11. Cf. M. Midgley: art. cit., pp. 34-35.

12. Para trazar la distinción entre dos tipos de semejanza me apoyo en las indicaciones que da Plotino, Eneadas, I, 2,2, cuando trata el problema de las virtudes. La indistincion entre estos dos tipos de semejanza es la que provoca la calda en al tercer hombres en el Paménides de Platón 132 d-133 a. Ni en Platón ni en Plotino la argumentación tiene absolutamente nada que ver con cuestiones concernientes ni a la igualdad ni a los géneros. Sólo tomo la forma del argumento, porque la distinción trazada me parece esclarecedora para aplicarla del modo en que yo lo hago.

13. Cfr. M. Midgley; art cit., p. 34.

14. La autonomia es un rasgo que casi todas las teóricas aceptan como necesario para poder hablar de igualdad, en buena parte porque se suele considerar a las mujeres privadas de ella * por naturalezan, no solo en los planteos clásicos como el de Rousseau, sino en planteos feministas partidarias de la "ética del cuidado" como Gilligan o Chodorow. Sin embargo, el concepto de autonomía es bastante problemático, como lo han mostrado diferentes autoras, como L. Code; art. cit., pp. 54-61; Jean Grimshaw: «Autonomy and Identity in Feminist Thinking", en GriffithsWhitford (eds.), op. cit., pp. 90-108; Alison Assiter: "Autonomy and Pomography, ${ }_{x_{1}}$ en Ibid, pp. 58-71; M. Ifurnm: op. cit., p. 14. Dificilmente pueda calificarse de autónoma a una mujer que elija o actúe por propia decision si el principio de su acción está condicionado por las expectativas socialmente inscritas on el estereotipo femenino. Tampoco puede considerarse autónoma a la mujer que, por ocupar posturas en el espacio público, actúe sigujendo los patrones masculinamente deseables.

15. Cf. Celia Amoros: *Mujeres, feminismo y poders, en Actas del Seminario Mujer y Poder. Madrid, U.C. Madrid, 1989, pp. 5-28; \&Violencia contra las mujeres y pactos patriarcales", en $V$. Maqueira y $C$. Sánchez (comp): Violencia y sociedad patriarcal. Madrid, P. Iglesias, 1978. Quien se ha ocupado particularmente de la cuestión del poder, en una perspectiva vecina a la de Amoros, es Amelia Valcárcel en Sexo y filosofia. Sobre «mujer y upoder», Barcelona, Anthropos. 1991. 
16. Tomo el concepto de equivalencia de $A$. Del Re, art. cit., p. 21.

17. C. Amoros ha insistido en la necesidad del reconocimiento mutuo que debe darse entre quienes constituyen un espacio de jguales, de pares, un grupo y no una serie. Además de los trabajos citados y de los desarrollos de su conocida obra Hacia una critica de la razón patriarcal, Madrid, Anthropos, 1985, ver *Espacio de los iguales, espacio de la idénticas. Notas sobre poder y principio de individuacion, Arbor, 128 (1987), pp. 113 126, El nuevo aspecto de la polisw, La Batsa de la Medusa, 19-20 (1991), pp. 119-135, y "Hongos hobbesianos, setas venenosas", Mientras Tanto, 48 (enero-febrero, 1992).

18. Cf. F. Collin: art cit., p. 136, quien toma la expresión "sujet interloqués de J. Marion.

19. Rescato la idea de responsabilidad, sin por ello compartir la línea de pensamiento expuesta en la obra, de No creas tener derechos de la Librería de Mujeres de Milan, trad. cast: Cuadernos inacabados, Madrid, Horas y Horas, 1991. p. 182.

20. L. Code, art. cit., caracteriza la igualdad recumiendo a tres conceptos: autonomía, autoridad (que hemos retomado aqui) y androginia (que preferimos dejar de lado). Cristina Molina por su parte, sugiere que la igualdad entre los sexos buscada por el feminismo quiza deba ser construida como solidaridad, a la manera en que la entiende R. Rorty. Cf, C. Molina: «El feminismo en la crisis del proyecto ilustrados, Sistema, 99, p. 141 (noviembre, 1990).

21. Cf. Ibid., p. 132.

22. Cf. Daryl McGowan Tress: «Comment on Flax's "Posmodernism and Gender Relations in Feminist Theory" , Signs, 14 (1988) 1, p. 197.

23. Cf. F. Collin: art. cit., p. 139.

24. Cf. Ibid, p. 70 .

25. Cf. especialmente *El nuevo aspecto de la polis*, art. cit., p. 133 y *Hongos hobbesianos, setas venenosas", art. cit., pp. 65-66.

26. Cf. No creas tetter derechos, op. cit., pp. 178. 180, si bien no comparto las estrategias propuestas para dar solución al problema de hallar la mediación entre el ser mujer individua y el ser mujer.

27. Cf, F, Collin: art. cit., p. 134; C. Amoros: al nuevo aspecto de la polis», art. cit, p. 134 . En un sentido diferente también usan la idea de ambivalencia A. Del Re: art cit. p. 22 y Maria Luisa Boccia: wLa ricerca della differenza*, Democrazia e Dititto (supl. 10), Materiali e atti, 1 (1988).

28. Cf. A. Valcárcel: op. cit., p. 109; C. Amoros: El nuevo aspecto de la poliss art. cit, p. 134.

29. Para el significado de la expresion, cf. E: Gilson: *Regto Dissimilitudinis de Platón à Saint Bernard de Clairvaux", Mediaeval Studies (1947). pp. 108-130. No estoy usando acá la noción de "espacion en el mismo sentido en que la utiliza C. Amorós cuando habla del "espacio de los igualesn contraponiéndolo al sespacio de las idénticass en el artfculo antes citado.

30. Uso la noción de *alteración, como contrapuesta a salienación en el sentido que le da F. Collin, art. cit. Cf. especialmente pp. 134-135.

31. Aportes para una crítica de la teoría del género. Este trabajo, fruto de un interesante ejercicio de pensar en conjunto, fue realizado junto con Alicia Gianella, Ana Marfa Bach, Mara Luisa Femenias y Margarita Roulet y presentado a las I Jornadas Nacionales de Filosofía de la Universidad de Salta y II Congreso de la Aso clación Filosófica de la República Argentina en agosto de 1991. Una versión levemente modificada se halla en prensa en la revista Feminaria, 9 (octubre, 1992), con cl título *Filosofia y génerom.

32. Hustracith y feminismo, tesis doctoral ine dita, 1987, p. 380 .

33. Cf. Seyla Benhabib: aFeminism and Postmodernism; an Uneasy Alliance», Praxis International 11 (1991), 2, pp. 146-147. Benhabib sostiene que el posmodernismo ha producido una desventajosa «retirada de la utopias dentro del feminismo; aclara que no toma "utopian en su significado moderno, sino como eprincipio regulativo de esperanza», sin el cual se vuelve inpensable la moralidad y toda transformación radical: Como anhelo de lo "totalmente otro" (das ganz Andere), de lo que aún no es, tal pensamiento utópico es un imperativo moral-prácilico [...] Pues nosotras, como mujeres, tenemos mucho que perder si abandonamos la esperanza utopica en lo totalmente otros.- Ver también M. Humm: op. cit., Pp. 228-229.

34. El concepto de objeto virtual está tomado de $\mathrm{H}$.: Lefebvre La revolución urbana, trad. cast., Madrid, Alianza, 1972, especialmente p. 11. EI objeto virtual es pasible de un examen al que Lefebvre llama straducción*. Cf. nuestro trabajo citado en nota 31.

35. Cf. F. Collin: art cit, pp. 137-138.

36. Cf. A. Valcárcel: op. cit., p. 70: Y si, en efecto, no puede asegurar'se que la igualdad entre varones y mujeres nos haga mejores a todos, como fue la optimista presunción del sufragismo y el reformismo, debe resaltarse, kantianamente, que es mejor ella misma por la universalidad que comportan.

37. Como, por ejemplo, A. Del Re: art. cit., p. 26 y C. Amoros: $\mathrm{El}$ nuevo aspecto de la polis , especialmente p. 135, y *Hongos hobbesianos, setas venenosas, especialmente pp. 65-67. El concepto de "pactos entre mujeres como red de relaciones entre mujeres no tiene nada que ver con las relaciones de affidamento entre mujeres que propugnan las mujeres de la Librería de Milán en 
No creas tener derechos, op. cit. (ver especialmente pp. 13-19 y 157-163).

38. Cf. Linda Alcoff: "Cultural Feminism versus Post-structuralism: the Identity Crisis in Feminist Theoryn, Signs, 13 (1988), 3, pp. 473-497 (trad. cast en Feminaria, II [1989], 4); R. Braidotti: art cit. p. 42.

39. Cf. R. Braidotti; art. cit., pp. 38-42; A Del Re: art. cit. pp. 24-25; Henrietta L. Moore: Ancropología y feminismo, trad, cast., Valencia, Cátedra, 1991, pp. 217-228.

40. Ibid., p. 46 .

41. Se trata de la falacia de falsa generaliza- ción que comete Protarco al comienzo del Filebo de Platón. Una excelente explicación de esta falacia se halla en I. Crombie: Analisis de las doctrinas de Platón, trad. cast., Madrid, Alianza, 1963, vol. II, pp. 354-362. Sobre este punto me be octlpado en "División y dialéctica en el Fedro", Revista Latinoamericana de Filosofía. XVI (1990), 2 , pp. 157-158. La falacia de falsa generalización es desmontada por Platón también en la República $V$, cuando toca el tema de las mujeres guardianas. Sobre este aspecto of. mi artículo ${ }^{*}$ Justicia $y$ género en Platón, República $V_{*}$, Hiparquia, I (1988), pp. 35-42. 